\title{
Abandono terapêutico na terapia cognitivo-comportamental
}

\author{
Drop-out in cognitive-behavior therapy
}

Juliana da Rosa Pureza ${ }^{[a]}$, Margareth da Silva Oliveira ${ }^{[b]}$, Ilana Andretta ${ }^{[c]}$

\footnotetext{
${ }^{\text {a] }}$ Mestranda em Psicologia Clínica na Universidade do Vale do Rio dos Sinos (Unisinos), Porto Alegre, RS - Brasil, e-mail: julianapureza@yahoo.com.br

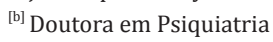
e Psicologia Médica pela Universidade Federal de São Paulo (Unifesp), professora do Programa de Pós-Graduação em Psicologia da Pontifícia Universidade Católica do Rio Grande do Sul (PUCRS), Porto Alegre, RS - Brasil, e-mail: marga@pucrs.br

${ }^{[c]}$ Doutora em Psicologia pela Pontifícia Universidade Católica do Rio Grande do Sul (PUCRS), professora do Programa de Pós-Graduação em Psicologia da Universidade do Vale do Rio dos Sinos (Unisinos), Porto Alegre, RS - Brasil, e-mail: ilana.andretta@gmail.com
}

Recebido: 13/07/2011 Received: 07/13/2011

Aprovado: 09/05/2012 Approved: 05/09/2012

\begin{abstract}
Resumo
O abandono terapêutico é um importante problema da na prática clínica, porém as conclusões que encontramos na literatura sobre o tema ainda são controversas, principalmente no que diz respeito ao abandono terapêutico na TCC. Dessa forma, o presente estudo tem como objetivo identificar que fatores estão relacionados com o abandono de pacientes na TCC em uma clínica-escola. Foram analisados os dados arquivados de 63 pacientes atendidos na TCC. As análises compararam os pacientes que abandonaram o tratamento e os que permaneceram no tratamento. A taxa de abandono observada foi similar ao reportado na literatura. Os pacientes que abandonaram o tratamento não diferiram dos que permaneceram em tratamento no que se refere à variáveis sócio-demográficas e ao diagnóstico. Os resultados indicaram que os pacientes que abandonaram o tratamento compareceram a um número menor de sessões, sugerindo que a maioria dos pacientes abandona a TCC nas sessões inicias. A literatura aponta que uma falha na boa relação terapêutica ou o uso de hipóteses terapêuticas equivocadas podem levar ao abandono nas sessões iniciais.
\end{abstract}

Palavras-chave: Pacientes que abandonam o tratamento. Terapia cognitiva. Psicologia clínica.

\begin{abstract}
The patient drop-out is a important problem we found in clinical practice, but the conclusions we found in literature about this subject are still controversial, especially as regards to understand drop-out in CBT. Because of this, this study had the objective to identify what factors are related to drop-out in CBT. Were analyzed archived data from 63 patients treated in CBT. The analyses compared patients who drop-out treatment and those who stay in treatment. The drop-out rate found was similar to literature's finds. Patients who drop-out treatment did not differ from patients who stay in treatment regards socio-demographic variables and diagnosis. The results indicate that patients who drop-out attended a fewer sessions, suggesting that the most patients leave the CBT in early sessions. The literature indicates that a break in therapeutic relationship or use of wrong therapeutic hypothesis can produce drop-out in early sessions.
\end{abstract}

Keywords: Patient dropouts. Cognitive therapy. Clinical psychology.

Psicol. Argum. 2013 jul./set., 31 (74), 561-568 


\section{Introdução}

Um importante problema que encontramos, tanto na prática quanto na pesquisa em psicologia clínica, é o abandono de pacientes do processo terapêutico. Esse abandono terapêutico pode ocorrer em diversas situações psicoterápicas, abrangendo não somente a psicoterapia individual em adultos, mas também a psicoterapia grupal, infantil, adolescente e a farmacoterapia (Beckham, 1992). 0 abandono de pacientes gera importantes implicações para a área da saúde mental, pois o custo da desistência de pacientes de tratamento em serviços de saúde é alto (Benetti \& Cunha, 2008; Luk et al., 2001; Salmoiraghi \& Sambhi, 2010). Graças a isso, nos últimos anos, diversos pesquisadores têm buscado entender o que leva as pessoas a desistirem de seus processos terapêuticos.

A literatura sobre o abandono de tratamentos em serviços de saúde mental é vasta, evidenciando a amplitude e severidade desse tópico. Os pesquisadores Wierzbicki e Pekarik (1993) realizaram um estudo de meta-análise sobre abandono em psicoterapia, onde o nível de abandono reportado nos estudos encontrados variou de $30 \%$ a $60 \%$. Em outros dois estudos brasileiros realizados nos últimos anos, a taxa de abandono também se aproximou de um terço dos pacientes atendidos (Melo \& Guimarães, 2005; Ribeiro, Alves, Vieira, Silva \& Lamas, 2008). Essa porcentagem de abandono que encontramos na literatura também é similar tanto para terapias individuais quanto para terapias grupais, sugerind o que nenhuma forma de terapia apresenta vantagem no que se refere ao abandono de pacientes (Davis, Hooke \& Page, 2006).

0 abandono terapêutico pode ser influenciado por diversas variáveis, como características do paciente, do terapeuta, da técnica e do setting de trabalho. 0 abandono abrange tanto atendimentos realizados na clínica privada como em serviços de saúde comunitária (Benetti \& Cunha, 2008). Por causa disso, o número de estudos visando a identificar as características do paciente e do tratamento associadas ao abandono terapêutico tem crescido na literatura. A maioria desses estudos tem como objetivo levantar e estimar prevalências de abandono, encontrar preditores de abandono e/ou descobrir variáveis correlacionadas, visando a propor estratégias que minimizem as altas taxas de evasão de tratamento (Gastaud, 2008). Os principais fatores associados ao abandono terapêutico encontrados na literatura são aqueles relacionados aos dados sociodemográficos e clínicos dos pacientes (Glombiewski, Hartwich-Tersek \& Rief, 2010; Hofmann \& Suvak, 2006; Melo \& Guimarães, 2005; Ribeiro et al., 2008) devido às dificuldades operacionais de se obter informações sobre a população que abandona (Melo \& Guimarães, 2005; Sales, 2003; Tehrani, Krussel, Borg \& Munk-Jorgensen, 1996).

$\mathrm{Na}$ meta-análise realizada por Wierzbicki e Pekarik (1993) foi identificado que as variáveis mais frequentemente associadas ao abandono terapêutico foram raça, nível educacional e renda. Alguns estudos também apontam que pacientes solteiros (Ribeiro et al., 2008; Tehrani et al., 1996), jovens (Arnow et al., 2007; Davis, Hooke \& Page, 2006; Melo \& Guimarães, 2005; Ribeiro et al., 2008; Tehrani et al., 1996) e desempregados (Ribeiro et al., 2008; Tehrani et al., 1996), com baixo nível socioeconômico e educacional (Salmoiraghi \& Sambhi, 2010) têm uma maior tendência em abandonar o tratamento psicoterápico. Não existe uma consistência entre o resultado dos estudos realizados no que se refere à diferença entre os sexos no abandono terapêutico. Com relação às variáveis clínicas dos pacientes que abandonam prematuramente o tratamento, a literatura aponta que a presença de um diagnóstico ou comorbidade psiquiátrica está associada ao abandono do tratamento, assim como a utilização de abordagem terapêutica exclusivamente farmacológica em comparação com o tratamento combinado (Ribeiro et al., 2008). No que se refere ao tipo de diagnóstico, o resultado dos estudos difere na identificação de um diagnóstico psiquiátrico mais freqüentemente presente em pacientes que abandonam (Salmoiraghi \& Sambhi, 2010). Isso ocorre porque muitas vezes acabam abandonando o tratamento sem ter um diagnóstico fechado, ou pelos estudos se centrarem apenas em algum tipo específico de diagnóstico, enquanto outros quadros psiquiátricos não são mencionados.

As variáveis relacionadas às características dos tratamentos aonde ocorre uma interrupção precoce do processo terapêutico têm sido frequentemente negligenciadas nos estudos sobre abandono (Tehrani et al., 1996). Diversos estudos realizados sobre este tema não mencionam qual o tipo de tratamento oferecido, o que dificulta a compreensão teórica do abandono terapêutico. Uma variável que parece ser preditora do abandono terapêutico é o alto número de faltas do paciente durante o processo de atendimento (Beckham, 1992), o que poderia servir como 
um sinal de aviso para o terapeuta, possibilitando uma intervenção no sentido de impedir o abandono.

A Terapia Cognitiva é uma abordagem terapêutica composta por diferentes protocolos de tratamento fundamentados no pressuposto de que não é a situação em si que causa o problema, mas sim o modo como o paciente interpreta a situação (Beck, 1997). 0 fato de esses protocolos de tratamento muitas vezes se utilizarem de técnicas comportamentais deu origem ao nome Terapia Cognitivo-Comportamental (TCC). A TCC foi desenvolvida nos anos 60, por Aaron, Temkin e Beck, a partir de insatisfações com as formulações psicodinâmicas para a depressão (Rangé \& Silvares, 2001). Atualmente, a TCC abrange diversas patologias psiquiátricas e é recomendada para o tratamento de conjunto amplo de distúrbios psicológicos e psiquiátricos. Apesar de inúmeros estudos comprovarem a eficácia da TCC para diversos transtornos (Almeida \& Neto, 2004; Salmoiraghi \& Sambhi, 2010), diversos pacientes abandonam essa modalidade de tratamento na prática clínica.

Muitas razões complexas são responsáveis pelas dificuldades que surgem durante o processo terapêutico da TCC, resultando em abandono. Essas razões podem estar relacionadas ao paciente, ao terapeuta e a outros fatores externos à sessão terapêutica (Beck, 2006). Segundo Bados, Balaguer e Saldaña (2007) os estudos mais recentes conduzidos em centros de saúde mental em diferentes países mostram que o índice de abandono varia entre $24 \%$ e $66 \%$, oscilando principalmente entre 35\% e $55 \%$. Outros estudos sobre TCC, no entanto, relatam uma porcentagem de abandono abaixo deste intervalo (Davis, Hooke \& Page, 2006; Glombiewski et al., 2010), sugerindo que ainda não existe um consenso quando buscamos compreender o índice de abandono de pacientes na TCC.

Essa dificuldade em se obter um consenso nos estudos sobre o abandono terapêutico pode ser explicada pela divergência na definição de abandono utilizada (Bados et al., 2007; Benetti \& Cunha, 2008; Glombiewski et al., 2010; Melo \& Guimarães, 2005; Keijsers, Kampman \& Hoogduin, 2001; Sales, 2003). Gastaud (2008) identificou em uma pesquisa realizada nas principais bases de dados eletrônicas que são utilizadas diversas definições de abandono terapêutico nos estudos sobre este tema, e que o uso de diferentes critérios pelos estudos torna impossível a comparação direta das taxas de abandono e dificulta a interpretação dos resultados encontrados na literatura. Além da definição de abandono utilizada, não há uniformidade nas situações e variáveis estudadas associadas ao abandono, variando no que se refere ao serviço prestado, à modalidade do tratamento, população utilizada, podendo apresentar ainda importantes diferenças culturais (Bados et al., 2007; Benetti \& Cunha, 2008; Glombiewski et al., 2010; Gastaud, 2008; Sales, 2003; Tehrani et al., 1996). Outra uma característica das pesquisas realizadas que dificultam a comparação entre os estudos é a utilização de amostras pequenas e heterogêneas (Keijsers et al., 2001).

Se existe uma dificuldade em realizar comparações entre os estudos realizados sobre abandono terapêutico de um modo geral, menos conclusivo ainda é o conhecimento científico acerca do abandono terapêutico na TCC, em virtude da pequena quantidade de estudos realizados sobre o tema nesta abordagem (Bados et al., 2007; Glombiewski et al., 2010; Salmoiraghi \& Sambhi, 2010). As diferenças encontradas entre os resultados dos estudos sobre abandono na TCC ainda dificulta o estabelecimento de medidas preventivas para o abandono deste tipo de tratamento. Esses dados são preocupantes, pois o abandono terapêutico, tanto na TCC quando em outras modalidades de terapia, parece ser ainda mais comum na prática clínica do dia a dia do que em pesquisas controladas (Bados et al., 2007). Graças à relevância desse assunto para a prática clínica da psicologia, e à necessidade do desenvolvimento de mais estudos acerca deste tema, o presente artigo tem como objetivo identificar que fatores estão relacionados com o abandono terapêutico de pacientes atendidos na abordagem cognitivo-comportamental em uma clínica-escola.

\section{Método}

Neste estudo, foi realizado um levantamento retrospectivo e quantitativo, utilizando os dados arquivados de pacientes atendidos no Serviço de Atendimento e Pesquisa em Psicologia (SAPP), clínica-escola da Faculdade de Psicologia da PUCRS, no período de março de 2006 a dezembro de 2009. Foram levantados os dados de 63 participantes, com idade entre 12 e 70 anos, que realizaram tratamento individual na abordagem cognitivo-comportamental no SAPP, no período de março de 2006 a dezembro de 2009. Essa amostra foi composta

Psicol. Argum. 2013 jul./set., 31 (74), 561-568 
tanto por participantes que abandonaram o tratamento psicoterapêutico como por participantes que permaneceram no tratamento até receberem alta e serem encaminhados para outros locais. Todos os pacientes cujos dados foram coletados já haviam finalizado o tratamento. A definição de abandono utilizada neste estudo foi de, após pelo menos um encontro com o terapeuta, o paciente não comparecer às consultas agendadas e não retornar para dar continuidade ao tratamento, apesar de ter sido convidado a fazê-lo. Essa definição foi baseada no estudo de Luk et al. (2001).

0 procedimento utilizado neste estudo para a coleta de dados consistiu em um levantamento do material dos pacientes que receberam tratamento no SAPP. O tratamento oferecido para os participantes consistiu em atendimento individual em psicoterapia breve na TCC. O SAPP é a clínica-escola vinculada à Faculdade de Psicologia da PUCRS, e oferece atendimento psicológico de baixo custo (conforme a renda) à comunidade, atingindo o público de Porto Alegre e região metropolitana. Conforme os procedimentos-padrão do SAPP, ao buscarem atendimento, os participantes passaram por um processo de triagem e, com base na deman$\mathrm{da}$, foram encaminhados para psicoterapia breve individual na abordagem cognitivo-comportamental, uma das modalidades de tratamento oferecidas. No processo de triagem, os participantes preencheram uma ficha de dados sociodemográficos do SAPP, que inclui informações gerais sobre os pacientes. Dentre as informações contidas nessa ficha, foram utilizados os seguintes dados para esta pesquisa: sexo, idade, escolaridade, estado civil, ocupação profissional, renda, genograma, data da triagem, realização ou não de psicodiagnóstico, data do primeiro atendimento, número de sessões e número de faltas. Todos os participantes incluídos na amostra assinaram o Termo de Consentimento Livre e Esclarecido padrão do serviço, permitindo que os dados de seus atendimentos fossem utilizados em atividades de pesquisa. Os atendimentos realizados foram conduzidos por estagiários do SAPP, e foram registrados em forma de relato ou dialogada, tendo sido supervisionados por um supervisor-professor da Faculdade de Psicologia da PUCRS.

Inicialmente, os dados foram tratados e analisados por meio da estatística descritiva. Para diferenciar o grupo de pacientes que abandonaram e o de pacientes que não abandonaram o tratamento, essa variável foi categorizada de forma dicotômica. Dessa forma, todas as análises descritivas foram realizadas por meio de tabulação cruzada, sendo o grupo colocado como uma das variáveis dessas análises. Essa análise inicial consistiu em descrever as características gerais da amostra, no que tange à média, desvios-padrão e frequência de dados categoriais ou ordinais. Em seguida, com a finalidade de identificar diferenças entre os grupos de abandono e não abandono, foram realizados testes $t$ de Student para amostras independentes (quando as variáveis eram numéricas continuas) e do qui-quadrado (para variáveis categóricas). As análises estatísticas contaram com um nível de significância $\mathrm{p} \leq 0,05$.

\section{Resultados}

Foi encontrado que, dos 63 pacientes incluídos na pesquisa $31,3 \%(n=20)$ eram do sexo masculino e $68,8 \%(\mathrm{n}=44)$ do sexo feminino. A média de idade dos participantes foi de 30,30 anos (DP = 1,858); a renda média foi de $\mathrm{R} \$ 811,16$ reais, entre 1 e 2 salários mínimos. A maioria dos pacientes $(98,4 \%)$ não relatou internação psiquiátrica nem psicodiagnóstico anterior ao atendimento. Dos 63 pacientes incluídos na amostra, 29 abandonaram o tratamento, totalizando uma taxa de abandono de 45,3\% da amostra.

Tendo em vista que o objetivo do estudo foi analisar a relação entre as variáveis sociodemográficas, clínicas e diagnósticas dos pacientes e o abandono de tratamento, a amostra foi dividida em pacientes que abandonaram o tratamento $(n=29)$ e pacientes que permaneceram em tratamento $(n=34)$. No grupo de pacientes que permaneceram em tratamento estão incluídos os pacientes que receberam alta do tratamento ou que foram encaminhados para outra instituição. A Tabela 1 ilustra a distribuição da amostra em relação às características sociodemográficas para variáveis categorizadas.

A comparação da idade e renda média entre os dois grupos não apresentou diferença estatística significativa, ( $p=0,785$ e $p=0,388)$, tendo o grupo que abandonou o tratamento apresentado uma idade média de 30,69 anos (DP $=15,455)$ e uma renda média de $\mathrm{R} \$ 916,55(\mathrm{DP}=877,98)$, enquanto o grupo que permaneceu em tratamento apresentou uma idade média de 29,65 anos (DP $=14,668$ ) e uma renda média de $\mathrm{R} \$ 734,82$ (DP = 781,02). Os dois grupos também se mostraram homogêneos no que se refere ao sexo $(p=0,681)$, escolaridade 
Tabela 1 - Características sociodemográficas da amostra

\begin{tabular}{llcccc}
\hline & & \multicolumn{3}{c}{ Grupo } \\
& & \multicolumn{2}{c}{ Abandonou } & \multicolumn{2}{c}{ Finalizou } \\
\hline \multirow{3}{*}{ Sexo } & Feminino & $\mathrm{n}$ & $\%$ & $\mathrm{n}$ & $\%$ \\
& Masculino & 21 & 72,4 & 23 & 67,5 \\
Escolaridade & Ensino Fundamental & 8 & 27,6 & 11 & 32,4 \\
& Ensino Médio & 7 & 24,1 & 8 & 23,5 \\
& Ensino Superior & 13 & 44,8 & 15 & 44,1 \\
Ocupação & Estudante & 9 & 31,0 & 11 & 32,4 \\
& Empregado & 13 & 44,8 & 12 & 35,3 \\
& Outros & 10 & 34,5 & 13 & 38,3 \\
Estado Civil & Sem companheiro (solteiro/separado) & 6 & 20,7 & 9 & 26,5 \\
& Com companheiro & 13 & 44,8 & 22 & 64,7 \\
Presença de filhos & Com filhos & 16 & 55,2 & 12 & 35,3 \\
& Sem filhos & 17 & 58,6 & 23 & 67,6 \\
& & 12 & 41,1 & 11 & 32,4 \\
\hline
\end{tabular}

Fonte: Dados da pesquisa.

( $p=0,994)$, ocupação ( $p=0,727)$, estado civil $(p=0,114)$ e presença de filhos $(p=0,458)$.

Não foram encontradas diferenças estatisticamente significativas no que se refere à presença de diagnóstico primário ( $\mathrm{p}=0,368$ ) e secundário ( $\mathrm{p}=0,174)$. Os diagnósticos utilizados foram os identificados pelos estagiários que realizaram o atendimento, de acordo com os critérios do Manual Diagnóstico e Estatístico de Transtornos Mentais (American Psychiatric Association, 2003). Também não houve diferença estatística no que diz respeito à medicação primária $(\mathrm{p}=0,682)$ e secundária $(\mathrm{p}=0,516)$. No que diz respeito à realização de atendimento psicológico prévio, também não houve diferenças entre os grupos $(p=0,550)$. A distribuição da amostra com relação a esses aspectos pode ser vista na Tabela 2 .

Com relação às características do atendimento clínico, os grupos diferiram no que se refere ao número de sessões realizadas $(p=0,001)$; o grupo que permaneceu em tratamento apresentou um número significativamente maior de sessões do que o que abandonou o tratamento, mas não diferiram com relação ao número de faltas $(\mathrm{p}=0,609)$ ou de dias na lista de espera $(p=0,673)$. 0 grupo que abandonou o tratamento apresentou média de 8,79 sessões (DP $=8,829)$ e de 3,55 faltas ( $\mathrm{DP}=2,995)$, tendo permanecido em média 147,03 dias (DP $=198,818$ ) na lista de espera, enquanto o grupo que permaneceu em tratamento apresentou uma média de 19,00 sessões (DP $=9,623$ ) e 3,18 faltas ( $D P=2,79)$, e permaneceu em média 127,18 dias $(D P=172,520)$ na lista de espera. Para descartar a hipótese de possível viés nos resultados devido aos dados de algum paciente específico, foi calculada a mediana do número de sessões realizadas para os dois grupos, de modo a identificar a medida de tendência central da amostra. 0 grupo de pacientes que abandonou o tratamento apresentou mediana de 6,0 sessões, enquanto o que permaneceu em tratamento apresentou mediana de 17,5 sessões, reforçando a ideia de que a maioria dos pacientes abandona o tratamento logo nas primeiras sessões.

\section{Discussão}

A taxa de abandono observada neste estudo $(45,3 \%)$ foi similar à reportada pela literatura clássica sobre abandono terapêutico (Wierzbicki \& Pekarik, 1993), nos resultados encontrados em estudos brasileiros recentes sobre o tema (Ribeiro et al., 2008; Melo \& Guimarães, 2005), bem como em outros estudos sobre abandono de pacientes na TCC (Bados et al., 2007). Isso pode sugerir que a taxa de abandono terapêutico na TCC não difere de outros tipos de terapia. As análises estatísticas realizadas (qui-quadrado, teste t) revelaram que

Psicol. Argum. 2013 jul./set., 31 (74), 561-568 
Tabela 2 - Características clínicas da amostra

\begin{tabular}{llcccc}
\hline & & \multicolumn{3}{c}{ Grupo } \\
& & \multicolumn{2}{c}{ Abandonou } & \multicolumn{2}{c}{ Finalizou } \\
\hline \multirow{3}{*}{ Diagnóstico Primário } & & $\mathrm{n}$ & $\%$ & $\mathrm{n}$ & $\%$ \\
& Sem diagnóstico & 5 & 17,2 & 6 & 17,6 \\
& Transtorno de Humor & 15 & 51,7 & 12 & 35,3 \\
& Outros diagnósticos de Eixo I & 9 & 31,0 & 16 & 47,1 \\
Diagnóstico Secundário & Com diagnóstico secundário & 8 & 27,6 & 15 & 44,1 \\
& Sem diagnóstico secundário & 21 & 72,4 & 19 & 55,9 \\
Uso de medicação primária & Usa medicação & 16 & 55,2 & 17 & 50,0 \\
& Não usa medicação & 13 & 44,8 & 17 & 50,0 \\
Uso de medicação secundária & Usa medicação & 8 & 27,6 & 7 & 20,6 \\
& Não usa medicação & 21 & 72,4 & 27 & 79,4 \\
Realização Prévia de Terapia & Já havia realizado terapia & 9 & 31,0 & 13 & 38,2 \\
& Nunca havia realizado terapia & 20 & 69,0 & 21 & 61,8 \\
\hline
\end{tabular}

Fonte: Dados da pesquisa.

as características demográficas da amostra dos pacientes que abandonaram o tratamento e dos que permaneceram no atendimento não apresentaram diferenças significativas.

Por outro lado, os pacientes que abandonaram o tratamento compareceram a um número significativamente menor de sessões do que os que finalizaram o tratamento. Bados et al. (2007) identificaram em seu estudo que a maioria dos pacientes que abandona a TCC faz isso nas primeiras sessões do tratamento, pois não encontraram o tipo de ajuda que procuravam ou não estabeleceram uma boa relação terapêutica. Com base nesses resultados, duas hipóteses para o abandono terapêutico precoce dos pacientes deste estudo serão exploradas.

Apesar de não ter sido possível avaliar essa variável neste estudo, a relação terapêutica é um aspecto importante de qualquer tratamento psicoterápico (Horvath \& Symonds, 1991). A ruptura nesta aliança terapêutica é um fator que pode contribuir para o abandono de pacientes na TCC (Bados et al., 2007; Beck, 2006; Glombiewski et al., 2010). Dessa forma, a relação terapêutica é uma variável que tem sido investigada em estudos sobre abandono terapêutico. A literatura aponta que ela se estabelece nas primeiras sessões da TCC, sendo fundamental para a manutenção do tratamento graças ao trabalho colaborativo realizado (Bados et al., 2007; Beckham, 1992). Alguns estudos indicam que uma fraca aliança terapêutica e insatisfação no início do tratamento estão relacionadas com o abandono precoce de pacientes (Arnow et al., 2007; Glombiewski et al., 2010; Piper, Ogrodniczuk, Joyce \& Mccallum, 1999). Dessa forma, o sucesso do tratamento dependerá da habilidade do terapeuta em avaliar, identificar e solucionar esse problema nas sessões iniciais, antes que resulte em desistência por parte do paciente (Lambert \& Barley, 2001). Uma das hipóteses para o abandono terapêutico dos pacientes deste estudo é a dificuldade no estabelecimento da relação terapêutica no inicio do tratamento, resultando no abandono precoce da TCC.

Apesar de a literatura apontar que a TCC tende a ter resultados terapêuticos relativamente rápidos, é comum nos depararmos com o abandono precoce de pacientes na TCC. Muitas vezes, quando os pacientes apresentam uma resposta pobre ou tardia no tratamento da TCC, isso pode significar que a dupla terapêutica está trabalhando com hipóteses terapêuticas equivocadas (Beck, 2006). Isso pode ocorrer por falha no processo de avaliação do paciente, que ocorre nas primeiras sessões da terapia e é fundamental para determinar a trajetória mais eficiente e efetiva do tratamento, pois sem ela o tratamento se torna vago ou irrelevante (Beck, 1997; Beck, 2006; Falcone, 2001). Além disso, a realização de uma avaliação correta do paciente permite o estabelecimento de uma relação terapêutica positiva nas sessões iniciais e uma maior adesão dele ao tratamento, fazendo com que o 
paciente se sinta compreendido e aceito (Lambert \& Barley, 2001; Rangé \& Silvares, 2001).

Um fator que pode ter contribuído para o abandono terapêutico precoce dos pacientes deste estudo é a realização de uma avaliação errônea ou incompleta do paciente, derivada da falta de experiência dos terapeutas que, por serem estagiários, podem ter tido dificuldades de compreender o funcionamento do paciente, mesmo sendo supervisionados. Essa dificuldade de terapeutas pouco experientes na avaliação do paciente pode fazer a terapia tomar uma direção não indicada e o paciente não encontrar o que ele precisava no tratamento, contribuindo também para um enfraquecimento da relação terapêutica e para o abandono do tratamento nas sessões iniciais.

Uma limitação encontrada neste estudo foi a dificuldade de se manipular variáveis de interesse, uma vez que o delineamento da pesquisa foi retrospectivo. Outra limitação do estudo realizado foi o tamanho da amostra utilizada, visto que a literatura sobre abandono terapêutico indica que a utilização de amostras pequenas e heterogêneas, como a desse estudo, dificulta a comparação com outros estudos já realizados sobre esse tema (Keijsers et al., 2001). O tamanho da amostra utilizada pode ter enviesado o estudo, impossibilitando encontrarmos diferenças significativas entre os grupos, quanto às outras variáveis analisadas neste estudo. Além disso, a procura por variáveis isoladas associadas ao abandono psicoterapêutico não leva a resultados consistentes, exigindo cada vez mais delineamentos de investigação voltados para a interação entre essas diversas variáveis, tais como a relação entre o terapeuta e o paciente (Lambert \& Barley, 2001; Piper et al., 1999).

\section{Considerações finais}

A literatura. Identificar e teorizar a respeito dos motivos que levam os pacientes a abandonar o tratamento nas sessões iniciais é fundamental para o desenvolvimento de estratégias de prevenção e retenção de pacientes no início do tratamento, aumentando a eficácia dos tratamentos cognitivo-comportamentais, produzindo maior qualidade de atendimento para os pacientes da TCC. Além disso, é importante que mais estudos sobre o abandono na TCC sejam realizados utilizando amostras robustas e homogêneas. É necessário realizar estudos que utilizem delineamentos experimentais e que levem em consideração a interação entre as variáveis e entre os participantes, de modo a complementar os resultados deste estudo, produzindo maior compreensão científica acerca do abandono terapêutico.

\section{Referências}

American Psychiatric Association (2003). Manual diagnóstico e estatístico de transtornos mentais. (4. ed.). Porto Alegre: Artmed.

Almeida, A. M., \& Neto, F. L. (2004). Indicações e contra-indicações. In P. Knapp (Org.). Terapia cognitivo-comportamental na prática psiquiátrica. Porto Alegre: Artmed.

Arnow, B., Blasey, C., Manber, R., Constantino, M., Markowitz, J., Klein, D., Thase, M., Kocsis, J., \& Rush J. (2007). Dropouts versus completers among chronically depressed outpatients. Journal of Affective Disorders, 97(1-3), 197-202.

Bados, A., Balaguer, G., \& Saldaña, C. (2007). The efficacy of cognitive-behavioral therapy and the problem of dropout. Journal of Clinical Psychology, 63(6), 585-592.

Beck, J. S. (1997). Terapia cognitiva: Teoria e prática. Porto Alegre: Artmed.

Beck, J. S. (2006). Indicações de problemas no tratamento. In J. S. Beck (Org.). Terapia cognitiva para desafios clínicos: $O$ que fazer quando o básico não funciona. Porto Alegre: Artmed.

Beckham, E. E. (1992). Predicting patient dropout in psychotherapy. Psychotherapy, 29(2), 177-182.

Benetti, S. P. C., \& Cunha, T. R. S. (2008). Abandono de tratamento psicoterápico: Implicações para a prática clínica Arquivos Brasileiros de Psicologia, 60(2), 48-59.

Davis, S., Hooke, G. R., \& Page, A. C. (2006). Identifying and targeting predictors of drop-out from group cognitive behaviour therapy. Australian Journal of Psychology, 58(1), 48-56.

Falcone, E. (2001). Psicoterapia Cognitiva. In B. Range (Org.). Psicoterapias cognitivo-comportamentais: Um diálogo com a psiquiatria. Porto Alegre: Artmed.

Gastaud, M. B. (2008). Abandono de tratamento na psicoterapia psicanalítica: Em busca de definição. Dissertação de Mestrado em Psicologia Clínica, Pontifícia Universidade Católica do Rio Grande do Sul, Porto Alegre. 
Glombiewski, J. A., Hartwich-Tersek, J., \& Rief, W. (2010). Attrition in cognitive-behavioral treatment of chronic back pain. Clinical Journal of Pain, 26(7), 593-601.

Hofmann S. G., \& Suvak M. (2006) Treatment attrition during group therapy for social phobia. Journal of Anxiety Disorders, 20(7), 961-972.

Horvath, A. O., \& Symonds, B. D. (1991). Relation between a working alliance and outcome in psychotherapy: A meta-analysis. Journal of Counseling Psychology, $38(2), 139-149$.

Keijsers, G. P. J., Kampman, M., \& Hoogduin, C. A. L. (2001). Dropout prediction in cognitive behavior therapyfor panic disorder. Behavior Therapy, 32(4), 739-749.

Lambert, M. J., \& Barley, D. E. (2001). Research summary on the therapeutic relationship and psychotherapy outcome. Psychotherapy, 38(4), 357-361.

Luk, E., Staiger, P., Mathai, J., Wong, L., Birleson, P., \& Adler, R. (2001). Children with persistent conduct problems who dropout of treatment. European Child and Adolescent Psychiatry, 10, 28-36.

Melo, A. P. S., \& Guimarães, M. D. C. (2005). Factors associated with psychiatric treatment dropout in a mental health reference center. Revista Brasileira de Psiquiatria, 27(2), 113-118.

Piper, W. E., Ogrodniczuk, J. S., Joyce, A. S., \& Mccallum, M. (1999). Prediction of dropping out in timelimited, interpretive individual psychotherapy. Psychotherapy, 36(2), 114-122.
Rangé, B., \& Silvares, E. F. M. (2001). Avaliação e formulação de casos clínicos adultos e infantis. In B. Rangé (Org.). Psicoterapias cognitivo-comportamentais: Um diálogo com a Psiquiatria. Porto Alegre: Artmed.

Ribeiro, M. S., Alves, M. J. M, Vieira, E. M. M., Silva, P. M., \& Lamas, C. V. D. (2008). Fatores associados ao abandono de tratamento em saúde mental em uma unidade de nível secundário do Sistema Municipal de Saúde. Jornal Brasileiro de Psiquiatria, 57(1), 16-22.

Sales, C. (2003). Understanding prior dropout in psychotherapy. Revista Internacional de Psicología y Terapia Psicológica, 3(1), 81-90.

Salmoiraghi, A., Sambhi, R. (2010). Early termination of cognitive-behavioural interventions: Literature review. The Psychiatrist, 34, 529-532.

Tehrani, E., Krussel, J., Borg, L., \& Munk-Jorgensen, P. (1996). Dropping out of psychiatric treatment: A prospective study of a first-admission cohort. Acta Psychiatrica Scandvica, 94(4), 266-271.

Wierzbicki, M., \& Pekarik, G. (1993). A meta-analysis of psychotherapy dropout. Professional Psychology: Research and Practice, 24(2), 190-195. 